\title{
Non-linear joint parameter identification using the frequency response function of the linear substructure
}

\author{
W-J Kim ${ }^{1 *}$, B-Y Lee ${ }^{1}$ and Y-S Park ${ }^{2}$ \\ ${ }^{1}$ School of Mechanical and Automotive Engineering, Keimyung University, Daegu, Korea \\ ${ }^{2}$ Center for Noise and Vibration Control, Department of Mechanical Engineering, Korea Advanced Institute of \\ Science and Technology, Daejeon, Korea
}

\begin{abstract}
A method based on frequency domain approaches is presented for the non-linear parameter identification of a structure having non-linear joints. The frequency response function (FRF) of the linear substructure, which can be calculated from the finite element method or measured by an experimental method, is used to calculate its FRFs needed in the parameter identification process. This method is easily applicable to a complex real structure having non-linear joints since it uses the FRF of the substructure. Since this method is performed in the frequency domain, the number of equations can be easily increased to as many as required to identify unknown parameters, not only by just varying the excitation amplitude but also by selecting the excitation frequencies. The validity of this method was tested numerically and experimentally with a cantilever beam having a non-linear element. It was verified through examples that the proposed method is useful to identify the non-linear joint parameters of a structure having arbitrary boundaries.
\end{abstract}

Keywords: non-linear parameter identification, frequency response function (FRF), linear substructure

\section{NOTATION}

$\boldsymbol{X}(\omega)$
$\mathbf{H}(\omega)$
$\boldsymbol{F}(\omega)$
$\boldsymbol{G}(\omega)$
$\{g(t)\}$
$g_{i j}(t)$
$\beta_{i j}$
$m$
$N$

$\mathbf{H} \boldsymbol{G}(\omega)$

Q

$P$

$\Delta f$

$\Delta F_{\mathrm{o}}$ response vector

the FRF matrix of substructure

external force vector

non-linear joint force vector

non-linear joint force vector

the $j$ th assumed joint force model of the $i$ th joint

the $j$ th model parameter of the $i$ th joint

the number of non-linear joints

the number of unknown model

parameters

the matrix obtained from multiplying the element of $\mathbf{H}_{22}(\omega)$ by that of $\boldsymbol{G}(\omega)$.

$(2 m \times N)$ real matrix in parameter

identification equation

$(N)$ real vector in parameter identification equation

frequency resolution

the amplitude increment of external force

The MS was received on 12 January 2004 and was accepted after revision for publication on 4 June 2004.

* Corresponding author: School of Mechanical and Automotive Engineering, Keimyung University, 1000 Shindang-dong, Dalseo-gu, Daegu 704-701, Korea.E-mail:wjkim@kmu.ac.kr
$A, B$
$h_{\mathrm{ee}}(\omega)$
$F_{\text {d }}$
excitation frequency components extracted from the non-linear response the point receptance of the beam at the free end Coulomb friction force

\section{INTRODUCTION}

The exact parameters of a model are necessary to analyse the behaviour of a mechanical system, but it is very difficult to determine its parameters using only theoretical methods. Experimental methods to identify the parameters therefore are generally used and many studies have been executed in this field. In particular, many mechanical systems are composed of some parts connected to one another by various types of non-linear joint. In this case, even if the dynamic model of each part is fairly accurately known and all the subsystems are quite linear, the whole system behaves non-linearly and the dynamic analysis of the whole system becomes complicated. Masri and co-workers [1-4] suggested a non-parametric method to identify non-linear joint properties. They constructed a joint model with the Chebyshev polynomial, taking advantage of its ortho- 
gonality characteristics, and fitted the model with the time domain states of the joint coordinate (displacement, velocity and acceleration). Recently Pacheco and Steffen [5] proposed a parametric methodology to identify the physical parameters of non-linear systems, through orthogonal functions. Also Crawley and Aubert [6] and Crawley and O'Donnell [7] identified the non-linear joint properties by plotting non-linear joint forces versus the states of the joint coordinates, called the 'force-state mapping technique'. This forcestate mapping technique is very simple and effective if the examining joint can be separated easily from the whole structure to be analysed. Lee and Park [8] proposed an efficient method to identify the positions and types of non-linear joint. They introduced a local identification method to identify joint properties using information about the positions of non-linear joints. However, most proposed methods have some limits when applied to an actual system because usually it is not easy to isolate the joint from the whole structure or because a large amount of response data is required due to time domain process. A frequency domain identification method, which is feasible to apply to actual systems, has been proposed by two of the present authors [9]. The advantage of the frequency domain method over the other methods is its reduction in the number of data points used in the final curve fit and the capability of identifying the parameters of a non-linear joint without testing the isolated joints. This work illustrates further developments enabling identification of systems with various types of non-linear joint including dry friction and the applications to actual structures through example studies to be addressed. The present method was applied numerically and experimentally to a cantilever with a non-linear joint. In numerical simulation, the method was tested with a cantilever beam characterized by the three types of nonlinear joint: velocity squared damping, cubic stiffness and dry friction. Also an experimental application to another cantilever with dry frictional force, which varies with its vibration modes, is presented. Numerical and experimental studies of cantilevers demonstrate the feasibility and utility of the present method.

\section{THEORETICAL FORMULATION}

When dividing the whole non-linear structure at the positions of non-linear joints, several linear substructures are generated. The frequency response functions (FRFs) of linear substructures can be easily obtained by using the finite element model or an experimental method. Assuming that the FRFs of each linear substructure are known, then the non-linear joint properties can be identified using the FRFs of linear substructures connected to the non-linear joint degrees of freedom. The effects of the non-linear joints can be treated as external forces by separating the substructures as shown in Fig. 1 and then the governing equation of motion of substructure in the frequency domain becomes

$$
\boldsymbol{X}(\omega)=\mathbf{H}(\omega)[\boldsymbol{F}(\omega)+\boldsymbol{G}(\omega)]
$$

where

$$
\begin{aligned}
\boldsymbol{X}(\omega) & =\text { response vector } \\
\mathbf{H}(\omega) & =\text { FRF matrix of substructure } \\
\boldsymbol{F}(\omega) & =\text { external force vector } \\
\boldsymbol{G}(\omega) & =\text { non-linear joint force vector }
\end{aligned}
$$

When dividing the FRF matrix of substructure into two parts depending on whether the degrees of freedom are directly connected to the joint or not, equation (1) can be rewritten as

$$
\begin{aligned}
\left\{\begin{array}{l}
\boldsymbol{X}_{1}(\omega) \\
\boldsymbol{X}_{2}(\omega)
\end{array}\right\}= & {\left[\begin{array}{ll}
\mathbf{H}_{11}(\omega) & \mathbf{H}_{12}(\omega) \\
\mathbf{H}_{21}(\omega) & \mathbf{H}_{22}(\omega)
\end{array}\right] } \\
& \times\left\{\begin{array}{l}
\boldsymbol{F}_{1}(\omega)+\boldsymbol{G}_{1}(\omega) \\
\boldsymbol{F}_{2}(\omega)+\boldsymbol{G}_{2}(\omega)
\end{array}\right\}
\end{aligned}
$$

where $\boldsymbol{X}_{1}(\omega)$ and $\boldsymbol{X}_{2}(\omega)$ refer to coordinates not

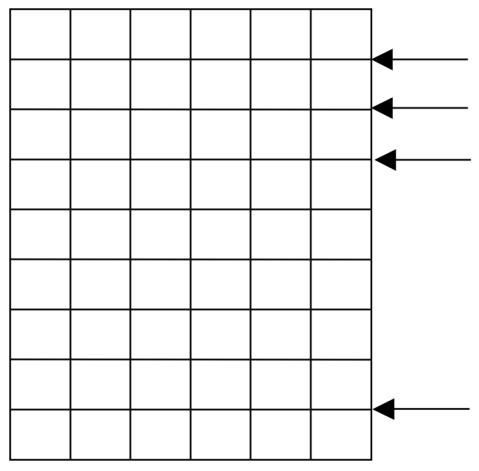

FE model

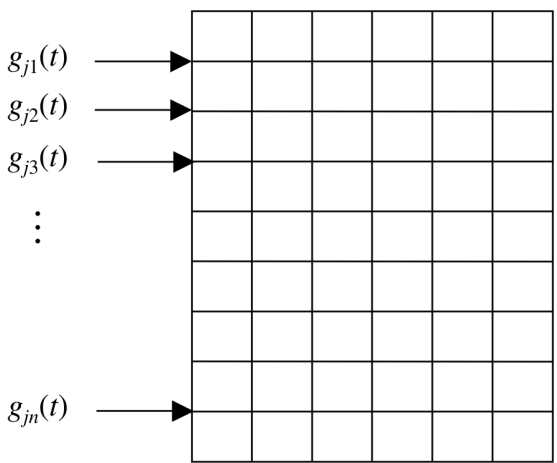

FE model

Fig. 1 Finite element (FE) model having non-linear joints 
connected and connected respectively to the joints. The joint forces at the coordinates, $\boldsymbol{G}_{1}(\omega)$, which are not connected to joints, become zero vector:

$$
\boldsymbol{G}_{1}(\omega)=\mathbf{0}
$$

The equation of the coordinates having joint forces is extracted from equation (2) as

$$
\begin{aligned}
\mathbf{H}_{22}(\omega) \boldsymbol{G}_{2}(\omega)= & \boldsymbol{X}_{2}(\omega)-\mathbf{H}_{21}(\omega) \boldsymbol{F}_{1}(\omega) \\
& -\mathbf{H}_{22}(\omega) \boldsymbol{F}_{2}(\omega)
\end{aligned}
$$

The right-hand side of equation (4) consists of the FRF of the substructure, responses and external forces at the positions which connected with joints. These values can be obtained from the results of analysis or experiment. It is convenient to denote the right-hand side of equation (4) by $\boldsymbol{R}(\omega)$ so that

$$
\mathbf{H}_{22}(\omega) \boldsymbol{G}_{2}(\omega)=\boldsymbol{R}(\omega)
$$

Since the joint force vector $\boldsymbol{G}_{2}(\omega)$ is only related to the joint responses, it can be represented as the functions of $\boldsymbol{X}_{2}(\omega)$ by introducing a model of non-linear joints. Assuming that $m$ joint forces are connected in the substructure, a joint force vector in the time domain becomes

$$
\{g(t)\}=\left\{\begin{array}{ccc}
g_{11}(t) \beta_{11}+ & \cdots & +g_{1 n_{1}}(t) \beta_{1 n_{1}} \\
g_{21}(t) \beta_{21}+ & \cdots & +g_{2 n_{2}}(t) \beta_{2 n_{2}} \\
& \vdots & \\
& \vdots & \\
g_{m 1}(t) \beta_{m 1}+ & \cdots & +g_{m n_{m}}(t) \beta_{m n_{m}}
\end{array}\right\}
$$

where

$g_{i j}(t)=j$ th assumed joint force model of the $i$ th joint

$\beta_{i j}=j$ th model parameter of the $i$ th joint

$m=$ number of non-linear joints

$N$ is the number of unknown model parameters that can be counted as follows:

$$
N=n_{1}+n_{2}+\cdots+n_{m}
$$

The joint force vector $\{g(t)\}$ can be determined from the joint force model of equation (6) if the responses of the joint coordinates, displacements and velocities are known. In order to complete an equation of parameter identification in the frequency domain, the responses of joints should be substituted into equation (6), which must be Fourier transformed. Fourier transforming the joint force vector gives

$$
\{G(\omega)\}=\left\{\begin{array}{ccc}
G_{11}(\omega) \beta_{11}+ & \cdots & +G_{1 n_{1}}(\omega) \beta_{1 n_{1}} \\
G_{21}(\omega) \beta_{21}+ & \cdots & +G_{2 n_{2}}(\omega) \beta_{2 n_{2}} \\
& \vdots & \\
G_{m 1}(\omega) \beta_{m 1}+ & \cdots & +G_{m n_{m}}(\omega) \beta_{m n_{m}}
\end{array}\right\}
$$

Substituting equation (8) into equation (5) and rearranging yield a linear algebraic set of equation to be solved for the unknown parameters $\beta_{i j}$, which can be written in a compact form as

$$
\mathbf{H} \boldsymbol{G}(\omega) \times \boldsymbol{\beta}=\boldsymbol{R}(\omega)
$$

where $\boldsymbol{\beta}$ is a vector expressed in terms of $\beta_{i j}$ and $\mathbf{H} \boldsymbol{G}(\omega)$ can be calculated by multiplying the element of $\boldsymbol{H}_{22}(\omega)$ by that of $\boldsymbol{G}(\omega)$. Since the elements of $\mathbf{H} \boldsymbol{G}(\omega)$ and $\boldsymbol{R}(\omega)$ are complex and those of $\boldsymbol{\beta}$ are real, then equation (9) can be divided into real and imaginary values such as

$$
\left[\begin{array}{c}
\operatorname{Re}[\mathbf{H G}(\omega)] \\
\operatorname{Im}[\mathbf{H} \boldsymbol{G}(\omega)]
\end{array}\right] \boldsymbol{\beta}=\left\{\begin{array}{l}
\operatorname{Re}[\boldsymbol{R}(\omega)] \\
\operatorname{Im}[\boldsymbol{R}(\omega)]
\end{array}\right\}
$$

where the numbers of unknown parameters and equations in equation (10) are $N$ and $2 m$ respectively. If $2 m \geqslant N$, then the unknown parameters $\beta_{i j}$ can be easily determined by the linear algebraic equation (10). The equations may be obtained by the sinusoidal excitation method which is popular to analyse the characteristics of the non-linear system. It is possible to make mutual independent equations by taking the joint coordinate responses for varying the amplitude or frequency of the sinusoidal excitation. A frequency variation method at a given excitation force level or an amplitude variation method at a given excitation frequency can therefore be adopted and those two methods can also be appropriately combined according to experiment conditions and the FRFs of substructures. When either $m$ frequencies or $m$ amplitude levels of excitation force are taken, then equation (10) will be expressed in the form

$$
\mathbf{Q} \boldsymbol{\beta}=\boldsymbol{p}
$$

where $\mathbf{Q}$ is a $2 m \times N$ real matrix and $\boldsymbol{p}$ is an $N$ real vector. Then the unknown parameter vector $\boldsymbol{\beta}$ can be determined by the pseudo-inverse technique:

$$
\beta=\left[\mathbf{Q}^{\mathrm{T}} \mathbf{Q}\right]^{-1} \mathbf{Q}^{\mathrm{T}} \boldsymbol{p}
$$

Finally, this procedure of the identification method of the non-linear joint parameters is described in a flow chart, as shown in Fig. 2.

\section{SIMULATION AND EXPERIMENT OF THE EXAMPLE SYSTEM}

\subsection{Simulation of the example system}

A cantilever beam having a non-linear joint at a free end, as shown in Fig. 3, is introduced to verify the nonlinear parameter identification method. Two types of non-linear joint model including velocity-squared damping, cubic stiffness and Coulomb friction are applied at the free end point of the cantilever beam. The beam is 


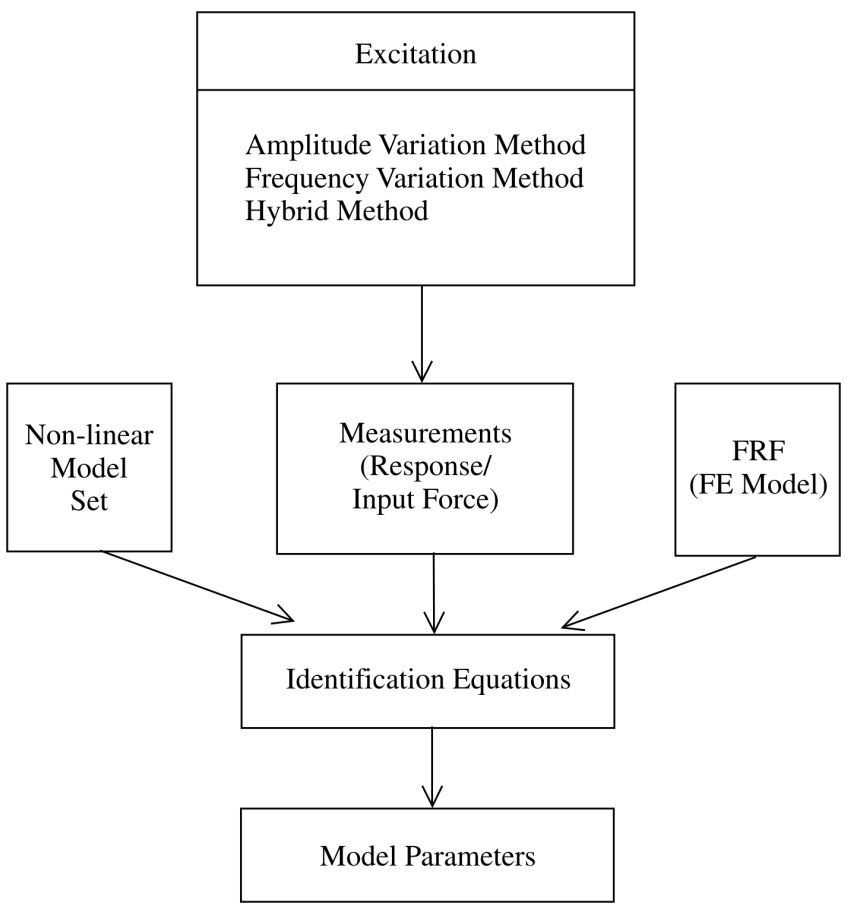

Fig. 2 Flow chart of parameter identification

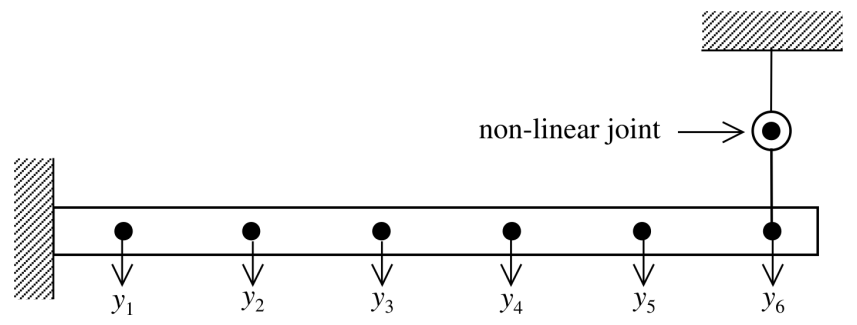

Fig. 3 Cantilever beam having a non-linear element

modelled as the finite element model, which has six elements with only vertical coordinates. The FRF of the beam is calculated by the finite element model without the non-linear joint at its end. Figure 4 shows the linear
Table 1 Model parameters of non-linear elements

\begin{tabular}{lllll}
\hline Type & $\beta_{1}(\mathrm{~N} / \mathrm{m})$ & $\beta_{2}(\mathrm{~N} \mathrm{~s} / \mathrm{m})$ & $\begin{array}{l}\beta_{3}\left(\mathrm{~N} \mathrm{~s}^{2} / \mathrm{m}^{2}\right) \\
(\mathrm{N})\end{array}$ & $\beta_{4}\left(10^{8} \mathrm{~N} / \mathrm{m}^{3}\right)$ \\
\hline Model I & 4800 & 20 & 500 & 1.6 \\
Model II & 4800 & 20 & 3 & 1.6 \\
\hline
\end{tabular}

FRF of the beam which represents a point receptance $h_{66}(\omega)$ at the free end of the beam, which has natural frequencies of 3 and $19 \mathrm{~Hz}$. The non-linear parameters of the joint models are identified in the neighbourhood of two resonance points in order to include sufficient non-linear effects and not to be sensitive to calculation errors. The following two types of non-linear joints are attached at the free end of the beam and then tested:

\section{Model I:}

$$
g(t)=y_{6} \beta_{1}+\dot{y}_{6} \beta_{2}+\dot{y}_{6}\left|\dot{y}_{6}\right| \beta_{3}+y_{6}^{3} \beta_{4}
$$

Model II:

$$
g(t)=y_{6} \beta_{1}+\dot{y}_{6} \beta_{2}+\frac{\dot{y}_{6}}{\left|\dot{y}_{6}\right|} \beta_{3}+y_{6}^{3} \beta_{4}
$$

where the given values of model parameters are shown in Table 1. Both joint models include a linear spring, viscous damping and cubic stiffness. In addition to those, one model has velocity-squared damping which represents a drag force in fluid and the other model has Coulomb friction which represents a dry frictional force. When the end point of the beam having the non-linear joint is excited by a sinusoidal force, the excitation force and response of the point are measured for the parameter identification procedure. In this example, equation (4) can be obtained using point receptance as follows:

$$
h_{66}(\omega) G(\omega)=Y_{6}(\omega)-h_{66}(\omega) F(\omega)
$$

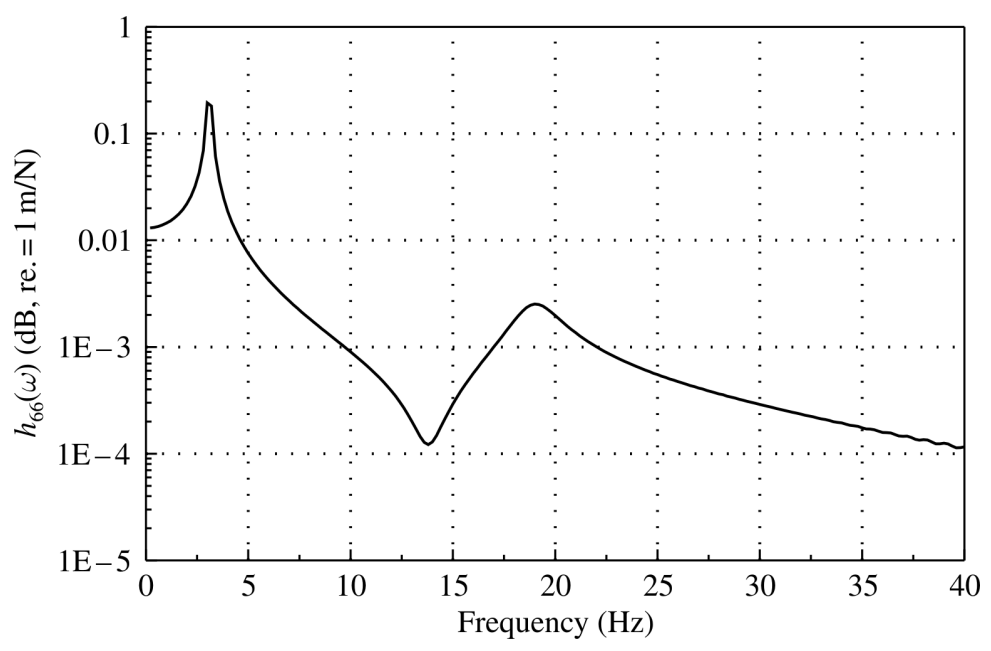

Fig. 4 Point receptance $h_{66}(\omega)$ of the cantilever beam 
where $G(\omega)$ is the non-linear joint force and $F(\omega)$ and $Y_{6}(\omega)$ are the excitation force and response respectively at the end point of the beam. Substituting the measured responses $y_{6}$ and $\dot{y}_{6}$ into the joint-model equation (13) or (14) and Fourier transforming both sides of that equation then yield $G(\omega)$, the non-linear joint force in the frequency domain, which includes the unknown parameters $\beta_{1}, \beta_{2}, \beta_{3}$ and $\beta_{4}$. Equation (15) can therefore be expressed in terms of the unknown parameters by substituting $G(\omega)$ into equation (15). Since this equation has complex values, it gives two real equations to identify the unknown parameters.

In order to obtain four parameters $\beta_{1}, \beta_{2}, \beta_{3}$ and $\beta_{4}$, the number of equations should be greater than four. As explained in the previous section, it is possible to increase the number of equations by varying the amplitude $F_{\mathrm{o}}$ or frequency $f$ of the sinusoidal excitation force as follows:

$$
f(t)=F_{\mathrm{o}} \sin (2 \pi f t)
$$

The following four cases, which are selected with varying amplitude or frequency of sinusoidal excitation force in the neighbourhood of the first mode and second mode natural frequencies are applied to increase the number of equations:

Case I: frequency variations in the vicinity of the first mode when $F_{\mathrm{o}}=80 \mathrm{~N}$ :

$$
f=1.0,1.4, \ldots, 4.6 \mathrm{~Hz}(\Delta f=0.4 \mathrm{~Hz})
$$

Case II: frequency variations in the vicinity of the second mode when $F_{\mathrm{O}}=80 \mathrm{~N}$ :

$$
f=17.0,17.4, \ldots, 20.6 \mathrm{~Hz}(\Delta f=0.4 \mathrm{~Hz})
$$

Case III: amplitude variations at the first mode natural frequency of $3 \mathrm{~Hz}$ :

$$
F_{\mathrm{o}}=10,20, \ldots, 100 \mathrm{~N}\left(\Delta F_{\mathrm{o}}=10 \mathrm{~N}\right)
$$

Case IV: amplitude variations at the second mode natural frequency of $19 \mathrm{~Hz}$ :

$$
F_{\mathrm{o}}=10,20, \ldots, 100 \mathrm{~N}\left(\Delta F_{\mathrm{o}}=10 \mathrm{~N}\right)
$$

This gives 20 equations in each case and the unknown parameters can be determined respectively by the leastsquares technique. These four cases are applied to the

Table 2 Identified parameters of model I

\begin{tabular}{lllll}
\hline Case & $\beta_{1}(\mathrm{~N} / \mathrm{m})$ & $\beta_{2}(\mathrm{~N} \mathrm{~s} / \mathrm{m})$ & $\beta_{3}\left(\mathrm{~N} \mathrm{~s}^{2} / \mathrm{m}^{2}\right)$ & $\beta_{4}\left(10^{8} \mathrm{~N} / \mathrm{m}^{3}\right)$ \\
\hline Case I & 4799.6 & 20.003 & 499.95 & 1.6003 \\
Case II & 4800.0 & 20.063 & 499.68 & 1.5998 \\
Case III & 4800.0 & 20.000 & 500.00 & 1.6000 \\
Case IV & 4800.0 & 20.000 & 499.99 & 1.5998 \\
Exact value & 4800 & 20 & 500 & 1.6 \\
\hline
\end{tabular}

C01504 (C) IMechE 2004 two non-linear joint models, models I and II respectively, and the results of identified parameters are summarized in Tables 2 and 3 according to model types and excitation cases.

The results shown in Tables 2 and 3 indicate that this method has a good performance to identify the nonlinear parameters of velocity-squared damping, cubic stiffness and Coulomb friction as well as the linear parameters of spring and viscous damping. The errors of model II in the results of identification are higher than those of model I because the Coulomb friction has a limit that cannot represent its dynamic behaviour exactly at zero velocity owing to discontinuity of model function. However, when analysing Table 3, even for the model including Coulomb friction, the identified results can be considered acceptable.

\subsection{Experiment of the beam structure}

The identification method was tested with a cantilever having Coulomb friction in order to verify and illustrate its validity and applicability to a real system. In the simulation of the example beam modelled with the finite element method, there are some errors in the identification of the joint model including dry friction even though they were not serious. Therefore this experimental work focused on the identification of the Coulomb friction parameter. A polyethylene cantilever beam, whose FRF can be easily measured because it has a relatively large damping, was used to estimate Coulomb friction. The length, width and thickness of the beam are $600 \mathrm{~mm}, 30 \mathrm{~mm}$ and $10 \mathrm{~mm}$ respectively. A thin plate spring was attached at the free end of the beam to generate a continuous frictional force as the beam oscillates as shown in Fig. 5. When conducting the experiment, special care was given to let the beam oscillate continuously and not to give stick-slip motion. The excitation amplitude and force were carefully adjusted to keep a constant frictional force. An exciter, which has a sufficient power to vibrate the beam structure with a given dry frictional force, is used. Even though the beam is well excited without dry friction by a sinusoidal excitation force, once the dry friction is loaded on the beam, the effects of friction distort the signal shape of excitation force. Therefore, it is necessary to control an excitation input in order to

Table 3 Identified parameters of model II

\begin{tabular}{lllll}
\hline Case & $\beta_{1}(\mathrm{~N} / \mathrm{m})$ & $\beta_{2}(\mathrm{~N} \mathrm{~s} / \mathrm{m})$ & $\beta_{3}(\mathrm{~N})$ & $\beta_{4}\left(10^{8} \mathrm{~N} / \mathrm{m}^{3}\right)$ \\
\hline Case I & 4707.3 & 20.040 & 2.9806 & 1.6226 \\
Case II & 4740.2 & 20.068 & 2.9192 & 1.6146 \\
Case III & 4754.2 & 20.631 & 2.9205 & 1.6108 \\
Case IV & 4821.8 & 20.054 & 2.9784 & 1.5940 \\
Exact value & 4800 & 20 & 3 & 1.6 \\
\hline
\end{tabular}

Proc. Instn Mech. Engrs Vol. 218 Part C: J. Mechanical Engineering Science 


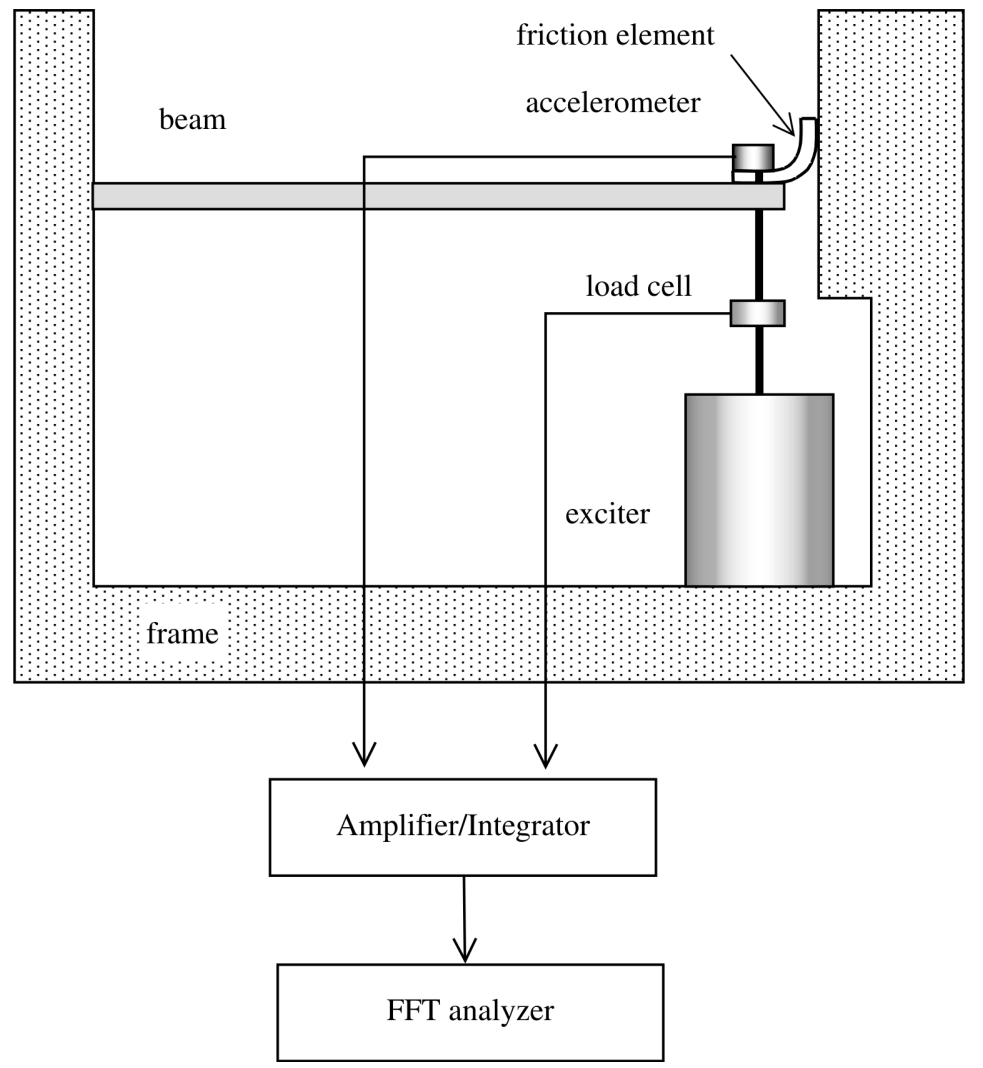

Fig. 5 Experimental apparatus (FFT, fast Fourier transform)

keep a pure sinusoidal force or to extract only the frequency component of excitation from the input force and response by signal filtering. For experimental convenience, the latter method was used to obtain the excitation force and the response for parameter identification. When extracting the excitation frequency component $F_{\mathrm{e}}(\omega)$ from the non-linear response, a fitting method using sine and cosine functions was directly applied to time domain data so as to reduce the errors of signal processing as follows:

$$
F_{\mathrm{e}}(\omega)=A-\mathrm{j} B
$$

where

$$
\begin{aligned}
& A=\frac{2}{N_{\mathrm{p}}} \sum_{n=1}^{N_{\mathrm{p}}} x(n) \cos (n \omega \Delta t) \\
& B=\frac{2}{N_{\mathrm{p}}} \sum_{n=1}^{N_{\mathrm{p}}} x(n) \sin (n \omega \Delta t)
\end{aligned}
$$

where $N_{\mathrm{p}}$ is the number of measured data points and $\Delta t$ is the sampling interval.

The linear FRF of the beam was measured from the cantilever without friction at its end. Figure 6 shows the linear point receptance $h_{\mathrm{ee}}(\omega)$ at the free end of the beam and its coherence function. Once the FRF was obtained, the frequencies were selected to give a good parameter estimation by examining the measured FRF. As shown in equation (12), the method may be sensitive to extraneous noise because it uses inverse calculation of a matrix that is made by multiplying the linear FRF and the non-linear functions of the response together. Therefore it is proper to select the frequencies in the vicinity of resonances, which have larger signal-to-noise ratios $(\mathrm{S} / \mathrm{N})$. Five frequencies of $28,29, \ldots, 32 \mathrm{~Hz}$ and $82,83, \ldots, 86 \mathrm{~Hz}$ which were near the second and fourth modes respectively of the beam were selected. The sign function was used for the Coulomb frictional force model:

$$
g(t)=F_{\mathrm{d}} \frac{\dot{x}}{|\dot{x}|}
$$

where $F_{\mathrm{d}}$ is the dry frictional force and, if $\dot{x}=0$, then $g(t)=0$.

The Coulomb force parameter $F_{\mathrm{d}}$ could be identified by using the linear FRF and the non-linear responses obtained via sinusoidal excitation. The main equation to identify the Coulomb force parameter can be written as equation (14) by using the measured FRF $h_{\mathrm{ee}}(\omega)$ :

$$
X(\omega)=h_{\mathrm{ee}}(\omega)\left[F_{\mathrm{e}}(\omega)+G(\omega)\right]
$$

where $F_{\mathrm{e}}(\omega)$ is the excitation force and $G(\omega)$ is the Coulomb frictional force. 


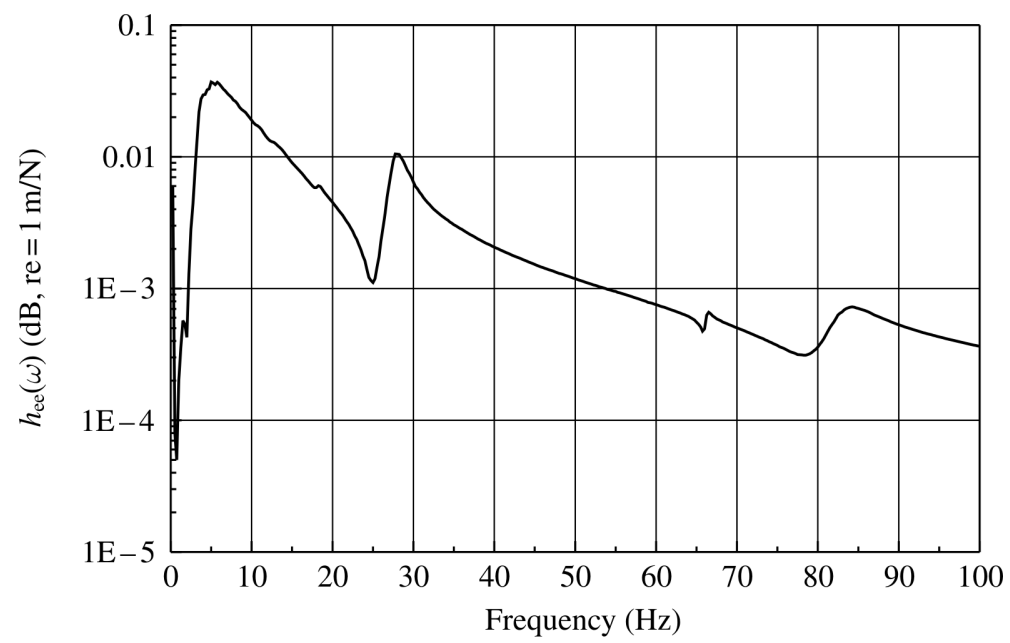

(a) Point receptance $h_{e e}(\omega)$

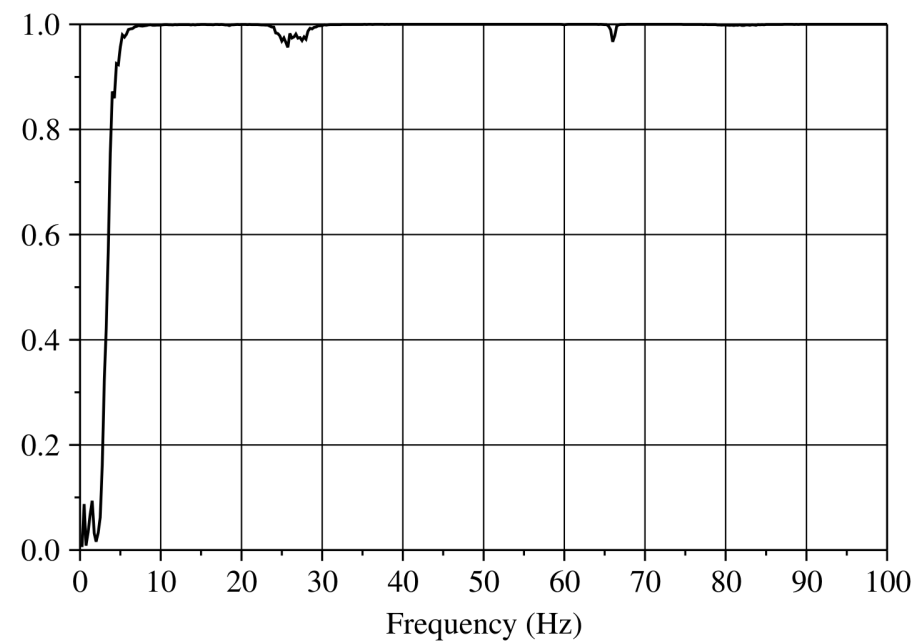

(b) Coherence

Fig. 6 (a) Point receptance at the free end and (b) its coherence

Two real equations to determine the Coulomb frictional force are obtained by dividing the complex equation (18) into two parts: real and imaginary. As mentioned above, since five frequencies in the vicinity of the second- and fourth-mode natural frequencies respectively of the beam were selected, ten real equations per mode were obtained. Table 4 lists the identified Coulomb parameters. By changing the excitation frequency, the Coulomb parameter was estimated. Also an optimum parameter was estimated by applying the least-squares method to ten real equations obtained from five different excitations. In Table $4, x_{\text {rms }}$ and $\dot{x}_{\text {rms }}$ denote the r.m.s. values of responses for displacement and velocity respectively that are used to estimate the Coulomb force. Analysing the results of Table 4, the Coulomb parameter obtained using the data around $30 \mathrm{~Hz}$ excitations (near the second mode) is quite different from the

C01504 C) IMechE 2004 value obtained from around the $84 \mathrm{~Hz}$ excitations (near the fourth mode). The difference can be explained because the beam tip oscillation is about three times larger $\left(X_{\mathrm{rms}}=2.756 \mathrm{~mm}\right.$ versus $\left.0.947 \mathrm{~mm}\right)$ when maintaining

Table 4 Identified results of Coulomb friction parameters

\begin{tabular}{|c|c|c|c|}
\hline \multicolumn{2}{|c|}{ Second mode } & \multicolumn{2}{|c|}{ Fourth mode } \\
\hline$f(\mathrm{~Hz})$ & $F_{\mathrm{d}}(\mathrm{N})$ & $f(\mathrm{~Hz})$ & $F_{\mathrm{d}}(\mathrm{N})$ \\
\hline 28 & 0.358 & 82 & 0.563 \\
\hline 29 & 0.349 & 83 & 0.616 \\
\hline 30 & 0.341 & 84 & 0.631 \\
\hline 31 & 0.336 & 85 & 0.598 \\
\hline 32 & 0.333 & 86 & 0.576 \\
\hline Optimum & 0.359 & Optimum & 0.602 \\
\hline \multicolumn{2}{|c|}{$\dot{x}_{\mathrm{rms}}=0.530 \mathrm{~m} / \mathrm{s}$} & \multicolumn{2}{|c|}{$\dot{x}_{\mathrm{rms}}=0.530 \mathrm{~m} / \mathrm{s}$} \\
\hline \multicolumn{2}{|c|}{$x_{\mathrm{rms}}=2.756 \mathrm{~mm}$} & \multicolumn{2}{|c|}{$x_{\mathrm{rms}}=2.756 \mathrm{~mm}$} \\
\hline
\end{tabular}

Proc. Instn Mech. Engrs Vol. 218 Part C: J. Mechanical Engineering Science 
about the same level of oscillating velocity. It is natural to say that a larger oscillation amplitude gives a smaller beam axial force and results in a smaller Coulomb frictional force. In order to verify the validity of the identified Coulomb parameter, the predicted response was compared with the measured value when a banded-random excitation force was applied. Figures 7 and 8 show the comparisons of the predicted and measured responses when the beam was excited by banded-random inputs of $12-44 \mathrm{~Hz}$ and $66-98 \mathrm{~Hz}$ respectively in the neighbourhoods of the second- and fourth-mode natural frequencies. From the above results, it can be observed that the identified Coulomb parameter predicts well the response of the beam, and therefore this method is feasible to identify the non-linear parameters.

\section{CONCLUSION}

A frequency domain method using the FRF of substructure to identify non-linear joint properties has been presented and tested numerically and experimentally with a cantilever having a non-linear joint. This method is characterized in that the joint forces, whether they are linear or non-linear, are treated as external forces and can be fitted using the substructure FRF and the joint responses in the frequency domain. In that sense, this method extends the force-state-mapping technique, which fits the joint forces with time domain joint degree response data, into frequency domain manipulations. Since this method is performed in the frequency domain, the number of equations required to identify the unknown parameters can be easily increased

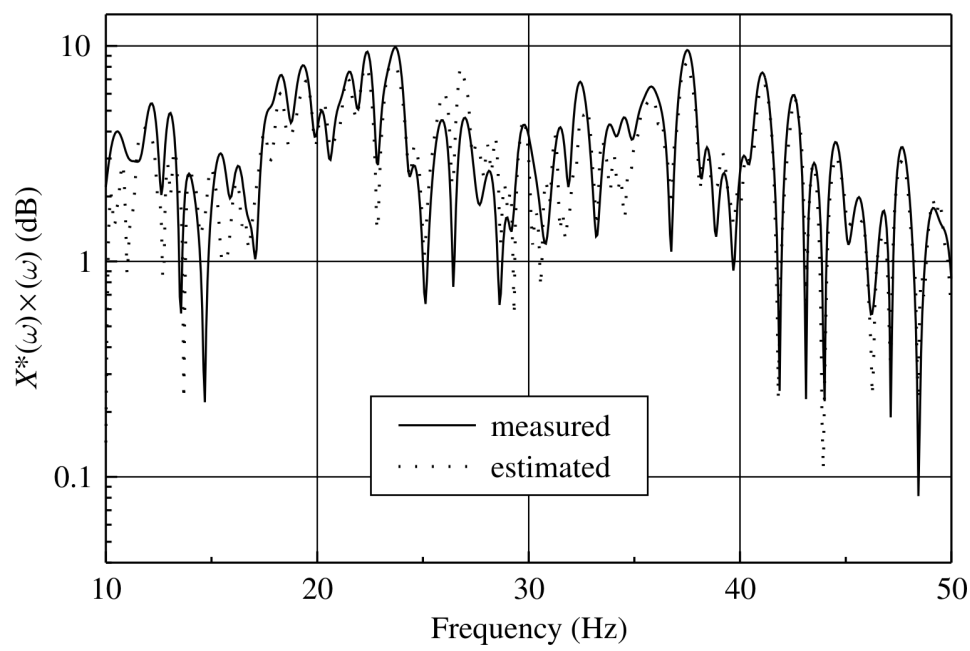

Fig. 7 The power spectra of measured and estimated responses when the input force has a frequency band of $12-44 \mathrm{~Hz}$

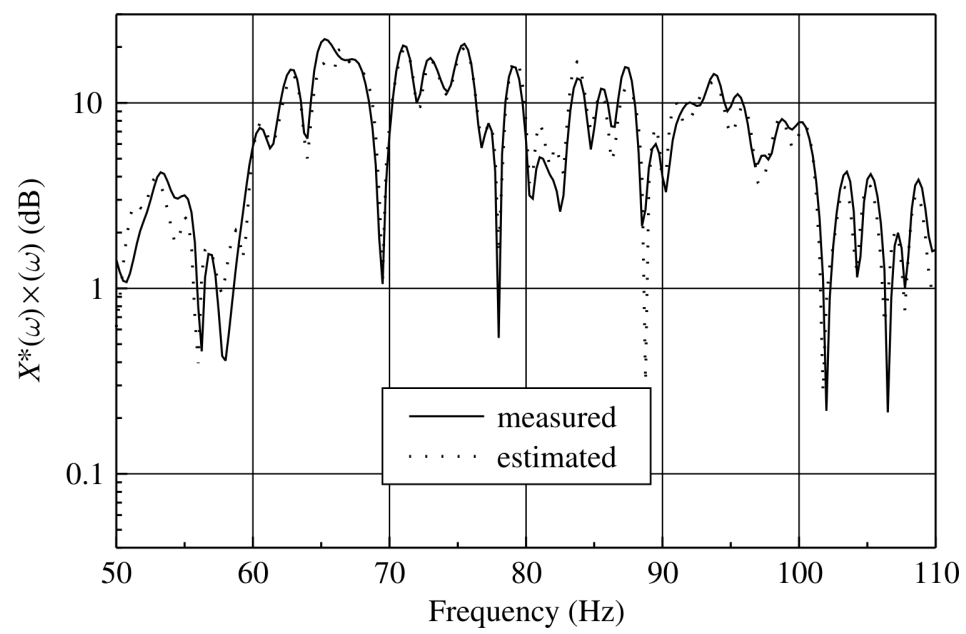

Fig. 8 The power spectra of measured and estimated responses when the input force has a frequency band of $66-98 \mathrm{~Hz}$ 
to as many as required in the procedure, by not only varying the excitation amplitude but also selecting excitation frequencies. It was verified through numerical and experimental examples that the method is useful to identify the non-linear parameters not only of a simple structure but also of a complex structure. This identification procedure is a simple scheme and can be applied to systems with different types of non-linearity and arbitrary non-linear boundaries, since it uses the FRF of substructure and the non-linear forces are treated as external forces.

\section{REFERENCES}

1 Masri, S. F. and Cauchey, T. K. A nonparametric identification technique for non-linear dynamic problem. Trans. ASME, J. Appl. Mechanics, 1979, 46, 433-447.

2 Masri, S. F., Sassi, H. and Caughey, T. K. Nonparametric identification of nearly arbitrary non-linear systems. Trans. ASME, J. Appl. Mechanics, 1982, 49, 619-628.
3 Masri, S. F., Miller, R. K., Saud, A. F. and Caughey, T. K. Identification of non-linear vibrating structures. Part I: formulation. Trans. ASME, J. Appl. Mechanics, 1987, 54, 918-922.

4 Masri, S. F., Miller, R. K., Saud, A. F. and Caughey, T. K. Identification of non-linear vibrating structures. Part II: application. Trans. ASME, J. Appl. Mechanics, 1987, 54, 923-929.

5 Pacheco, R. P. and Steffen Jr, V. On the identification of non-linear mechanical systems using orthogonal functions. Int. J. Non-linear Mechanics, 2004, 32, 1147-1159

6 Crawley, E. F. and Aubert, A. C. Identification of non-linear structural element by force-state mapping. Am. Inst. Aeronaut. Astronaut. J., 1986, 24(1), 155-162.

7 Crawley, E. F. and O'Donnell, K. J. Force-state mapping identification of non-linear joints. Am. Inst. Aeronaut. Astronaut. J., 1987, 25(7), 1003-1010.

8 Lee, S. S. and Park, Y. S. Position of non-linear element and identification of their type by a local non-parametric method. Mech. Systems Signal Processing, 1991, 5(5), 403-420.

9 Kim, W. J. and Park, Y. S. Non-linear joint parameter identification by applying the force-state mapping technique in the frequency domain. Mech. Systems Signal Processing, 1994, 8(5), 519-529. 
